\title{
The Factor Assay of Indicators of Physical and Functional Preparation of Basketball Players 18-25 Years Old
}

\author{
Dao Chanh Thuc*1, Hang Quang Thai ${ }^{2}$ and Doan Tien Trung ${ }^{3}$ \\ ${ }^{1,2}$ Deparment of Physical Education, AN GIANG University, Vietnam \\ ${ }^{3}$ Deparment of Physical Education, Ho Chi Minh City University of Education, Vietnam
}

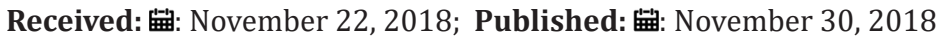

*Corresponding author: Dao Chanh Thuc, Deparment of Physical Education, An Giang University, Vietnam

\begin{abstract}
The problem of physiological study of non-traditional means of coaching attracts a lot of scientists' attention in recent years. This is due to the need to provide a maximum development of certain muscle groups and direction of the coaching process. Proper use of simulators weight machines bring positive results Valeria T et al. [1]. Obviously, an important step in the use of technology is the right choice for team sports coaches and personal trainers for the effectiveness of the coaching process. One effective component of this process is the inclusion to a coaching program with the help of physical exercises would be TRX® function loops. Among specialists of functional and physical coaching innovation as TRX®-coaching is one of the best equipment for complex solution of the tasks. This exercise is an exercise using body weight. It was designed to develop strength, power, core stability, flexibility and balance. TRX® is the valuable creation for the sports industry of any levels. TRX@ Coaching-suspension Coaching became commonly usable for Fitness Health Clubs, Professional Sports and Elite Strength Conditioning Workout for Sports Teams Dao CT et al. [2]. Therefore, it was suggested the use of TRX® exercises in preparation of basketball players. The results of the study clearly show that the inclusion in the 18-25 years old basketball player coaching program with the TRX® functional loop contributes to a significant increase in overall fitness levels and ability to foster gas of life. Using TRX® during coaching can be considered to be an important factor in increasing the effectiveness of basketball preparation.
\end{abstract}

Keywords: Basketball; Coaching Process; Objective; Method

\section{Introduction}

Suspension Coaching (TRX®) is considering as the best option for both beginners and professional athletes because it's easy to operate and manage. For example, Nicholas Ratamess describes the body weight exercises that are highly popular and the suspension coaching or total body weight resistance exercise (TRX®), that is used in school- and community-based programs to track progress, develop personalized programs, provide motivation, and assess the effectiveness of a fitness program Ratamess et al. [3]. In addition, working with elderly participants, many of whom with multiple chronic injuries, and required supervision to create modification that could help them maintain and improve performance without aggravating any preexisting conditions, Suspension Coaching was introduced into their regular coaching routines Gaedtke et al. [4]. Further, the research was conducted, using TRX®, among 36 health but untrained men. Scientists were focused on exercises that could improve strength, power, speed and jumping ability. However, after the study was completed, the scientists determined there was no significant improvement Maté-Muñoz et al. [5].

In effect, very few scientists have compared the effects of balance on performance measures in coaching programs performed under both unstable and stable conditions Sparkes et al. [6]. Thus, exploring results of wrestlers 10-12 years old suggest that TRX® functional loops can be used in the coaching program, as this tool increases: strength, speed-strength performance muscles in the arms and legs. However, muscle growth, as indicated, is insignificant that is valuable for wrestlers who limited weight category Miroshnikov et al. [7]. During the research on the effectiveness of coaching for qualified handball players, changes were identified and aerobic physical performance of athletes for two leading microcycles with TRX® assistance to conduct four major exercises for the lower body (Humstring Runner, Side Lunge, Overhead Squats). This has led to positive dynamic Tyshchenko et al. [8]. However, in the study 
of a particular sport, such as basketball, scientists were focused on finding out if three weeks of TRX® suspension coaching were enough to enhance static balance in junior basketball players. Those three weeks of TRX® coaching seemed to be insufficient for modifications to occur in the static balance of junior players Iuliana et al. [9].

It is necessary to generate explosive muscular forces very rapidly to accelerate, change directions, and then reaccelerate over relatively short distances in Team Spots Dao CT et al. [2]. Therefore, maximal velocity is likely a greater predictor of success, and the ability to change velocity over short distances in multiple directions based on game- specific stimuli is prevailing Dawes et al. [10]. Because of that, athletes must apply greater muscular forces into the ground to overcome the oppositional forces of gravity to produce velocity Kovacs [11]. In addition, the assay of scientific methodic literature defied that there is a deficit of sport science and movement-specific functional resistance coaching data on strength and conditioning for basketball players at every level. Therefore, it requires a deep assay of every action would be taken and influenced on basketball. Recently, experts in various sports widely used factor assay technique that reveals the complex dominant components Tishchenko [12]. This causes the athletic performance, determines the nature of the relationship between the indicators. Also it allows determine the contribution of individual factors to the overall result. Relevant is the definition of the factor structure of preparedness in basketball, which is an extremely important task. However, the literature wasn't paying enough attention to this question. Establishing main components for preparedness would greatly enhance the effectiveness of their coaching and competitive activity.

\section{Materials \& Methods \\ Participants:}

The study involved 26 basketball players between the ages of 18 to 25 years old team of Vietnam.

\section{Goals and Procedures:}

The goal of this study was to use TRX® to determine the effect on the functional readiness for basketball players. To solve this goal, we have the following tasks:

i. Assess the level of physical and aerobic performance in a coaching process for basketball players 18-25 years old.

ii. Analyse the dynamics of physical condition and aerobic capacity of basketball player's primary and experimental groups.

iii. Collocate the leading factors of functional readiness for basketball players.

Overall Design: The coaching in both groups was within the base stabilizing mesocycle specially preparatory phase. The control group of 13 athletes was involved in accordance to the traditional coaching program. Conventional exercises that meet the stated objectives of each part were used. The experimental group of 13 engaged involving specially designed complex exercises using TRX® equipment. Each exercise was performed 45 seconds, break- 15 seconds, every round- by 2 sets. The break between sets -1 minute. The total duration of one set 7 minutes. The total duration of the coaching 45 minutes. The study was undertaken in Vietnam after approval by the Institutional Research Ethics Committee at HoChiMinh City National University. Participation was voluntary; participants received no incentives. They were provided with information about the aims, objectives and methods of the study before the start of monitoring.

Data was anonymous and confidential, and data protection was observed at all the time. Each basketball player gave voluntary informed consent for the inclusion in the study. Measuring methods. Estimated overall physical performance using the method Astranddetermined level of overall physical performance, aerobic power and capacity. In the beginning and at the end of the preparatory and competitive macrocycle periods of basketball players, both groups were assessed by level of physical capacity and aerobic performance using submaximal test PWC170. Exact and relative values were calculated PWC170 (aPWC170,kgm/min; rPWC170, kgm/min/ $\mathrm{kg}$ ) and maximal oxygen consumption (aMOC, l/min; rMOC, l/min/ $\mathrm{kg}$ ), coefficient of efficiency of blood supply (CEBS), the power index (PI), index hypoxia (IH). Statistical assay. Generalization of the studied charact eristics was assessed by mean arithmetic value, standard deviation, error of mean arithmetic and factor an analysis. Confidence of differences between mean values was stated by Student's t-criterion. Assessment of statistical hypothesizes based on $5 \%$ significance level. For statistical processing of data, we used licensed program (SPSS 20.0).

\section{Results}

Preliminary physical fitness testing of basketball players who participated in the study made it possible to establish the following. As seen from the results in early coaching in preliminary and experimental groups were characterized by substantially equal values and overall physical performance aerobic productivity (Table 1). Levels aPWC170, rPWC170, aMOC, and rMOC matched low value. In general, the pre-test results determined the insufficient level of physical fitness of the surveyed groups (Table 1). The conclusive evidence of the above results was the data relative changes in the study of physical performance and aerobic productivity for basketball players in the control and experimental groups. In the assay of the functional state of the body on the subject to maintain the overall physical performance, it can be stated that for the period of rest not the adaptive stereotypes embodied for the past seasons have been lost. Therefore, with an average energy at the beginning of the season, the leading role of aerobic mechanisms in central and vice versa could be saved, to ensure the efficiency of the anaerobic organism in point guards laid down as a result of the coaching process, as well as according to their physiological characteristics. As a matter of fact, the implementation of the coaching program leads to a significant increase in performance and efficiency of power supply arrangements for the players. It should be noted for preservation of specific stereotype for all players' positions in basketball. 
Table 1: Dynamics of indicators of functional preparation for basketball players 18-25 years old during the annual macrocycle.

\begin{tabular}{|c|c|c|c|c|c|c|c|c|c|}
\hline Indicators & BPP & EPP & $t_{B P P_{E P P}}$ & ВCP & ECP & $t_{\mathrm{BCP}_{\mathrm{ECP}}}$ & $t_{\mathrm{BPP}{ }_{\mathrm{BCP}}}$ & $t_{\mathrm{EPP}{ }^{\prime} \mathrm{BCP}}$ & $t_{\text {EPP' }}{ }_{\text {ECP }}$ \\
\hline \multicolumn{10}{|c|}{ Control Group } \\
\hline aPWC170 & $1559.7 \pm 22.81$ & $1614.4 \pm 26.63$ & 1.63 & $1619.72 \pm 25.64$ & $1564.3 \pm 26.1$ & 1.68 & 1.71 & 0.06 & 1.58 \\
\hline rPWC170 & $17.19 \pm 0.16$ & $17.39 \pm 0.19$ & 1.03 & $17.17 \pm 0.12$ & $17.09 \pm 0.13$ & 0.37 & 0.05 & 1.03 & 1.44 \\
\hline aMOC & $4548.8 \pm 59.25$ & $4683.7 \pm 49.95$ & 1.69 & $4708.08 \pm 60.22$ & $4536.61 \pm 64.32$ & 1.89 & 1.84 & 0.34 & 1.69 \\
\hline rMOC & $47.84 \pm 0.38$ & $51.34 \pm 0.63$ & 2.78 & $51.04 \pm 0.37$ & $48.97 \pm 1.24$ & 1.27 & 2.09 & 1.17 & 1.78 \\
\hline \multicolumn{10}{|c|}{ Experimental Group } \\
\hline aPWC170 & $1569.72 \pm 25.68$ & $1726,86 \pm 31,9$ & 3,51 & $1668,2 \pm 29,3$ & $1558,84 \pm 24,3$ & 2,87 & 2,22 & 1,35 & 4,19 \\
\hline rPWC170 & $17.14 \pm 0.16$ & $18.68 \pm 0.15$ & 6.43 & $18.07 \pm 0.19$ & $17.12 \pm 0.17$ & 4.83 & 4.18 & 2.37 & 6.19 \\
\hline aMOC & $4541.15 \pm 59.32$ & $4871.21 \pm 68.23$ & 3.49 & $4739.12 \pm 54.63$ & $4497.64 \pm 50.51$ & 2.73 & 2.21 & 1.27 & 4.33 \\
\hline rMOC & $48.65 \pm 0.37$ & $53.75 \pm 0.35$ & 6.71 & $52.91 \pm 0.36$ & $49.53 \pm 1.27$ & 1.78 & 4.61 & 2.38 & 2.71 \\
\hline
\end{tabular}

Note: BPP-the beginning of the preparatory period; EPP-the end of the preparation period; BCP - the beginning of the competition period; ECP - the end of the competition period.

Further, to summarize the last phase of the survey, the attention should be paid about the rising level of fatigue and the partial depletion of energy systems. It should be emphasized that this decline is not dramatic and disastrous nature of all the indicators of power supply is not reduced to a level that allows speak of exhaustion and inability to maintain physical condition of basketball players. The results obtained in all phases made it possible to determine the orientation of quality changes in the studied parameters during the basketball season, the quantitative characteristics and the possibility of correction in coaching and competitive process for greater optimization and an increase in the functional state of the organism. This tendency is to increase the total of all indicators of physical performance and energy mechanisms including the competitive level, maintaining the level of these indicators in the first half of the season and reduce them to the background processes, developing due to fatigue at the end of the season.

Notes: BPP- The beginning of the preparatory period; EPPthe end of the preparation period; $\mathrm{BCP}$ - the beginning of the competition period; ECP-the end of the competition period.
Therefore, participants of the experimental group during the study were characterized by a significantly higher magnitude aPWC170 gain values, rPWC170, aMOC and rMOC, basketball players than the control group (Table 1). According to the algorithm method of prime component, the accuracy of survey results is achieved if the percentage of the sample is substantially between interconnected elements is not less than $60 \%$ of the total variance. When analyzing the factorial study of functional readiness for basketball players, the structure value of mentioned values in all groups exceeds the desired range, which was 78\%. One of the key points of our study was to determine the relationship between the level of functional manifestations of athletes and the effectiveness of physical fitness using TRX®. The (Table 2) evidenced how interconnected TRX® certain exercises between each other, and the level of manifestation for functional readiness. Factor assay procedure allowed to generate the system load factor and determine the three factors of physical fitness, eigenvalues were higher than one (Table 2). In general, the composition factor (whose contribution to the total dispersion made $16.34 \%$ ) entered parameters which characterize TRX® exercise for the upper body: Roll up $r=0.868$; Table Row $r=$ 0.903; TRX® Twist $r=0.882$.

Table 2: The grouping and distribution of the elements of physical fitness for basketball players 18-25 years old by using the primary method component.

\begin{tabular}{|c|c|c|c|c|}
\hline \multirow{2}{*}{ No } & \multirow{2}{*}{ Components } & F1 & Factors & F2 \\
\cline { 3 - 5 } & & 0.321 & 0.647 & 0.777 \\
\hline $\mathrm{x} 1$ & $\mathrm{aPWC170}$ & 0.448 & 0.719 & 0.567 \\
\hline $\mathrm{x} 2$ & $\mathrm{aMOC}$ & 0.755 & 0.215 & 0.842 \\
\hline $\mathrm{x} 4$ & CEBS & 0.246 & 0.596 & 0.695 \\
\hline $\mathrm{x} 5$ & $\mathrm{PI}$ & 0.638 & 0.329 & 0.589 \\
\hline
\end{tabular}

Note: The factor loadings are significant $(\mathrm{p}=0.05)$.

Cite this article: Dao Chanh Thuc, Hang Quang Thai, Doan Tien Trung. The Factor Assay of Indicators of Physical and Functional Preparation of Basketball Players 18-25 Years Old. Biomed J Sci \& Tech Res 11(4)-2018. BJSTR. MS.ID.002124. DOI: 10.26717/ BJSTR.2018.11.002124. 
The second factor (20.37\%) clustered index, which reflected the TRX® exercises for the thighs and buttocks: TRX® Frog $r=$ 0.916; Sprinter Start $r=0.778$; Wide Jump $r=0.747$. The third factor $(41.26 \%)$ was above the threshold load variables that reflect TRX® exercise for the upper and lower body: Burpee $r=$ 0.925; Push up +Tuck Knee $r=0.865$; Mountain Climber $r=0.875$. Subsequent graphic assay of the influence factors on the selected parameters Ai ... Zi possible to determine it in the form of a bipartite graph (chromatic number is 2 ), since a plurality of vertices can be broken into two parts (A) and (Z), so that neither one vertex in (A) is not connected with vertices $(A)$ and no vertex in $(Z)$ with vertices (Z) (the subset of vertices (A) and (Z) called lobes bipartite graph $G)$. This clearly shows the effect of each factor on the performance aPWC170, aMOC, CEBS, PI, IH (Figure 1). The values of z1, z2 and $\mathrm{z} 4$ are determined by the combined effect of the factors A2 and A3 and the indexes $\mathrm{z} 3$ and $\mathrm{z} 5$ - the combined effect of $\mathrm{A} 1$ and $\mathrm{A} 3$. The graph of the optimization based on the weights of the vertex allows us to determine that the graph contains an Eulerian circuit: A3 - z1 - A2 - z2 - A3 - z5 - A1 - z3 - A3 - z4 - A2. (Figure 2). That confirms that the graph is connected (Euler's cycle if and only if the graph is connected) and, accordingly - the impact of the factors determined in the parameters studied. Optimized G graphing (VFX, E) allows to determine the peak of the graph vertices $\mathrm{kA} 3=4$, confirming the effect of A3 (TRX® exercise for the upper and lower) (Figure 2).

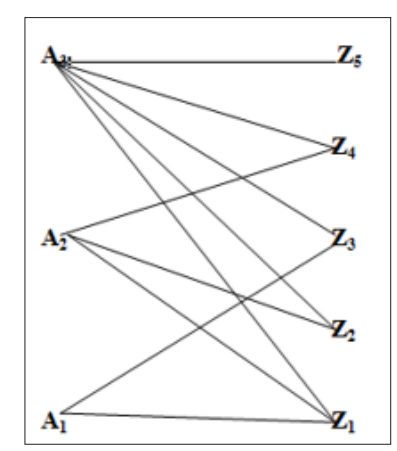

Figure 1: Bipartite graph G (VFX, E).

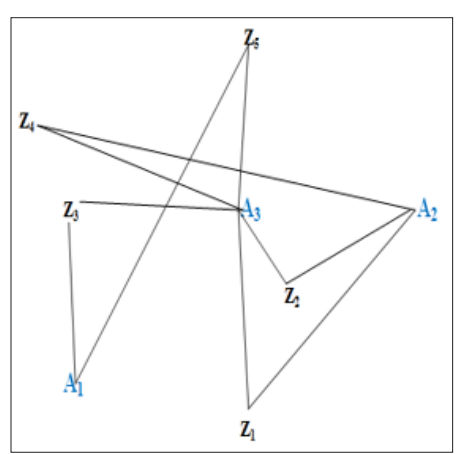

Figure 2: Graphical representation of the optimized graph $\mathrm{G}(\mathrm{VFX}, \mathrm{E})$ based on the weights of the vertices.

\section{Discussion}

A number of experts in the field of theory and methodology of sports coaching indicate that the modern technology of preparation of top-class athletes is based on the results of scientific research and has scientific and methodological platform Ratamess et al. $[3,2]$. Various scientists have developed biomechanical and psychopedagogical bases of an activity for athletes, as well as key aspects of their coaching: technical, physical, psychological and theoretical Kovacs et al. [11,2]. Thus, numerous studies suggest that a scientific approach to the coaching qualified athletes capable of providing high achievements Dao CT et al. [2]. Basketball is a dynamic and explosive sport, full of intensity and contacts Valeria T et al. [14]. That's the reason why a lot of basketball players can perform elements of the game, such as dunking the ball or quick movements through defenders and dribble to the net effortlessly. All those aspects take the huge influence on the athlete's body. With the use of Suspension Coaching exercises the player can simulate the way his body moves on the court, making him more able to handle challenges of practices and matches. By the variety of coaching and uneven load of work on the body, the TRX® Trainer better develops core strength and stability element to the required moves which can help the basketball players to be stronger and have better balance on the court Valeria $\mathrm{T}$ et al. [14]. In recent years, due to the increase of the struggle on the international arena of Vietnamese basketball teams, the issue of coaching competitive basketball players has gained a special meaning. Moreover, in the past several Olympic cycles formed the trend has been focused on increasing the system of complexity of technical and tactical action. To solve the problem and to achieve better results, the question has been awakened to use the advanced technology. In this regard, it is particularly important and urgent to train and prepare highly qualified basketball players. In particular, Vietnamese basketball is an urgent requirement to implement and implement higher complexity factors and their impact on competitive outcomes. They do not exist or cannot be included in the preparation of the structure depending on the coaching personnel.

The coach's main purpose of the practice is to concentrate on the players physical abilities developing their speed, strength and explosive power to make sure they have a good balance and able to jump high, etc. The biggest importance to joint stability is not necessarily strength or endurance but motor control. Instability resistance exercises promote co-contractions, increasing joint stability Behm et al. [13]. The idea is to involve the whole athlete's body during the practice, and how capable it is to challenge the ball and work under the net on the basketball court. TRX® allows the coach to create coaching programs for the players using progressing and regressing movements for strength, conditioning, stretching, rehabilitation, and to give additional bust to their bodies needed on scheduled practices or before competitions Valeria T et al. [1].

Suspension bodyweight coaching has many advantages. Moreover, many benefits it has brought to sports performance particularly in basketball. Jungle Gym, TRX®, Rotational Bodyweight Trainer from CrossCore $180 \AA$ helped bring this form of coaching to the forefront of the sports performance and fitness industries (Valeria. T et al, 2018). The latter of which has an added component of rotation with a greater equalization.

These are allgreatoptions that may increase an athlete's strength through a full range of motion, while utilizing a fully portable unit 
for convenience Hess et al. [14]. Using TRX® Coaching programs would benefit basketball players in order to achieve better balance and coordination on the both sides of the hardwood. The programs could vary from Beginner to Advance depending on the athletes' physical condition. TRX® assists the athletes to develop and perform basketball movements, concentrate on specific muscles and improve coaching outcomes. Based on these facts, several exercises TRX® are proposed that could be valuable as a part of the coaching program designed to increase preparedness of basketball players 18-25 years old.

\section{Conclusion}

The results of the study have clearly demonstrated that the introduction of training for basketball players with the TRX® function rings has contributed to a significant increase in overall fitness levels and ability to foster Body gas. Our research study element allows us to determine the presence of internal relationships between the indicators of the Super League's functional and physical preparation for the Vietnamese team. Obviously, the use of TRX® can be considered a factor to increase the efficiency of the training process. The main advantage - is to work with its own weight, without additional complications that allow the athlete to remove the vertical load. In the study, dynamics allows for accurate use, to make changes at all stages of the training process as a whole team and as individual players, who tend to higher.

\section{References}

1. Valeria T, Yaroslav H, Viktoria P, Oleksij Oleg B, Zoryana S (2018) Factor analysis of indicators of physical and functional preparation of basketball players. Journal of Physical Education and Sport 18(4): 1839-1844.

2. Dao Chanh Thuc, Le Thi Minh Dao (2018) Dynamics Of Changes In The Functional State Of Qualified Volleyball Players During Macrocycle. International Journal of Scientific \& Engineering Research 9(5): 17551759 .

\section{ISSN: 2574-1241}

DOI: 10.26717/BJSTR.2018.11.002124

Dao Chanh Thuc. Biomed J Sci \& Tech Res

This work is licensed under Creative

Commons Attribution 4.0 License

Submission Link: https://biomedres.us/submit-manuscript.php
3. Ratamess NA (2012) ACSM's foundations of strength training and conditioning. Wolters Kluwer Health/Lippincott Williams \& Wilkins pp. 229-232.

4. Gaedtke A, Morat T (2016) Effects of Two 12-week Strengthening Programmes on Functional Mobility, Strength and Balance of Older Adults: Comparison between TRX Suspension Training versus an Elastic Band Resistance Training. Central European Journal of Sport Sciences and Medicine 13(1): 49-64.

5. Maté Muñoz JL, Monroy AJA, Jiménez PJ, Garnacho Castaño MV (2014) Effects of instability versus traditional resistance training on strength, power and velocity in untrained men. Journal of sports science \& medicine 13(3): 460.

6. Sparkes R, Behm DG (2010) Training adaptations associated with an 8-week instability resistance training program with recreationally active individuals. Journal of Strength \& Conditioning Research 24(7): 1931-1941.

7. Miroshnikov A, Nesterov $\mathrm{P}$, Pashkin $\mathrm{P}$, Tabakov S, Myakinchenko E (2011) Using the training device TRX functional loops in the physical preparation of sportsmen-wrestlers 10-12 years. Lika pp. 117-121.

8. Tyshchenko VA (2014) The use of innovative technologies in educationtraining process of handballers. Visnyk of Chernigiv National Pedagogical University. S.: Pedagogical Sciences. Physical education and sports 118(4): 211-214.

9. Iuliana BB, Graniela Flavia DEAK, Simona M, Adrian P (2015) TRX suspension training method and static balance in junior basketball players. Educatio artis gymnasticae p. 27-34.

10. Dawes J, Lentz D (2012) Methods of developing power to improve acceleration for the non-track athlete. Strength \& Conditioning Journal 34(6): 44-51.

11. Kovacs M (2012) Training for Linear Acceleration. Colorado Springs, CO: NSCA Hot Topic Series.

12. Tishchenko VA (2016) Skilled handball player functionality variation in annual macrocycle. Theory and Practice of Physical Culture 3: 72-73.

13. Behm DG, Drinkwater EJ, Willardson JM, Cowley PM (2011) The role of instability rehabilitative resistance training for the core musculature. Strength \& Conditioning Journal 33(3): 72-81.

14. Hess S, Camacho C (2014) Rotational basketball movements. Strength Training 1(3): 22 .

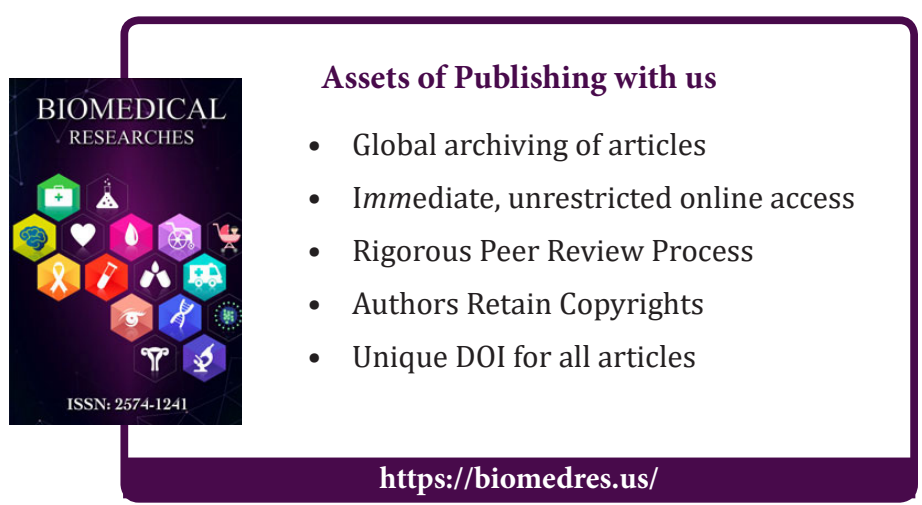

